\title{
Spectroscopic Imaging of Solid Planetary Surfaces
}

\author{
P.C. Pinet
}

UPR 234/CNRS, Observatoire Midi-Pyrénées, 31400 Toulouse, France

\section{Introduction}

Seen from Sirius through the eye of the telescope, our inner solar system would easily fit within one CCD-pixel. The purpose of the present paper is: i) to provide with a general overview of the use of imaging or 3D-spectroscopy for the study of the solid planetary surfaces, ii) to demonstrate that the analysis of 3D spectroscopic data on the basis of spectral mixture modelling permits to describe the subpixel spectral variability related to mineralogy of the planetary solid surfaces. In the following, a few cases are discussed concerning the remote sensing investigation in the UV-VIS-nIR domain of the lunar, terrestrial and martian surfaces, documented by means of multispectral or hyperspectral data, produced by telescopic, airborne or orbital imaging spectroscopic techniques.

\section{Background, Instrumentation and Methodology}

Advances in remote sensing technology have led to the development of imag. ing or mapping spectroscopy which can be used to map surface mineralogical variations. Mapping spectrometers measure both spatial and spectral information, producing the near-infrared reflectance spectrum for every picture element (pixel) in the image. The identification of a number of constitutive minerals present at the surface is made from the position, strength, and shape of absorption bands. As an example, pyroxenes are readily identifiable from their characteristic $\mathrm{Fe}^{2+}$ electronic transition absorption bands located near 1 and 2 microns while olivine is characterized by its wide asymmetrical absorption band around $1.05 \mu \mathrm{m}$. In the recent past, telescopic, orbital and airborne instrumentation based on different technologies has been successfully used in the field of planetary surface geology, including the study of the Earth, Moon and Mars. One simple but efficient alternative currently in use in the case of telescopic and orbital surveys of the Moon and Earth (e.g., T2M/Pic-du-Midi, CLEMENTINE mission, MERIS) is based on CCD multispectral imaging, using filter wheels associated with a limited number $(<10)$ of moderate bandpasses interferential filters $(R<100)$ ranging from 0.4 to 1.1 microns. In the case of Mars telescopic observations, more advanced technological solutions have been in use: i) the Wide Field Grism CCD spectrograph of the University of Hawaii 2.2m telescope in a slit-scanning mode (pushbroom technique), resulting in the production of 0.4-0.95 $\mu \mathrm{m}$ image cubes $(R=250-300)$. However, the quality of the image reconstruction only allowed for synoptic scale $(300-500 \mathrm{~km})$ studies of martian surface spectral variability (Bell et al. 1990), ii) the TIGER integral field spectrograph mounted on the $3.6 \mathrm{~m} / \mathrm{CFH}$ telescope at Hawaii to produce a mosaicked mapping 
of Mars, spanning the spectral domain $(0.85-1.05 \mu \mathrm{m})$ at resolution $R=600$, and with a spatial resolution $250-300 \mathrm{~km}$. Orbital observations of equatorial regions have also been obtained by the scanning spectrometer ISM aboard Phobos-2, covering the spectral range $0.76-3.1$ microns $(R=60$, spatial resolution: $20 \times 20$ $\mathrm{km}^{2}$ ). The two- dimensionality of the image of the surface is obtained by rotating the entrance mirror to scan in the cross track direction for the image samples and the forward motion of the spacecraft provides the image lines. For the purpose of terrestrial geology remote sensing studies, an airborne version of this instrument is also used. However the narrowness of the observed swath is a serious limitation for true imaging. This limitation is overcome by the NASA/AVIRIS (Airborne Visible/Infrared Imaging Spectrometer) instrument, with which the radiation collected by the foreoptics on a $512 \times 512$ CCD array, is carried along optical fibers to four spectrometers covering the range 0.4-2.45 $\mu \mathrm{m}$ $(R=100)$ (NASA 1987). Whatever the instrumental approach, one of the foremost problems with the production of hyperspectral images (3D-spectroscopy) is the data analysis and display. A new methodology, based on linear mixture modeling (Adams et al. 1986), is just gaining wide acceptance in the imaging of solid-surfaced planets and recent results presented here demonstrate its general power and interest. In particular, the spectral identification of constitutive minerals combined with this mixing analysis allows mineralogical contributions detected across a planetary surface to be measured and correlated with observed morphological units. It leads to detailed compositional and geological mappings.

\section{Results}

\subsection{Moon}

New Earth-based remote sensing techniques allow powerful investigations of the nature and layering of the lunar crust by means of spectro-imaging analyses, carried out at the subkilometer scale on the basis of the UV-visible-near infrared surface characteristics. Indeed, large impact craters such as Copernicus excavate materials from different depths and thus provide information at the target site on the preimpact stratigraphy and mineralogical heterogeneities of the lunar crust, both laterally and vertically. In particular, a detailed spectromineralogical map of the Copernicus interior and its surroundings is produced by applying a spectral mixing analysis (Pinet et al. 1993). This analysis shows that a mixing of three components can account for nearly all the total variance of the image. The distribution of the first endmember leads to a first-order mapping of the impact melt material within and around the crater. It constrains the volumetric amount of this material which has been generally underestimated in previous impact cratering modellings. The second endmember reveals a surface unit covering the outer part of the rim and locally contributing to the surface material on the southwestern walls and terraces of the crater. Its spectral properties might be characteristic of mare-like material. The third endmember relates to the presence of an olivine-bearing lithology component. It is detected not only within the three central peaks (Pieters \&Wilhelms 1985) but also along a significant portion of the crater rim. Consideration of an olivine-bearing endmember emphasizes the overall morphological pattern of the rim and wall terraces in the associated fraction image. These results suggest that the petrology of the tar- 
get was uniform throughout the stratigraphic horizons from which the rim and peak materials originated and the identification of widely exposed olivine units supports the idea that the lower crust and possibly the lunar mantle itself are regionally at shallow depth. Such an approach is also relevant to lunar geologic questions including the understanding of the crustal emplacement and evolution of the lunar basaltism, the role of the impact basins in the observed crustal stratigraphy (Head et al. 1993), the characterization of the physical properties of the regolith. The Clementine global spectral dataset, acquired from a lunar polar orbit ( $200 \mathrm{~m}$ spatial resolution), will be extremely useful in these matters.

\subsection{Mars}

Spectro-imaging telescopic observations (e.g., Pinet \& Chevrel 1990; Bell 1992; Martin et al. this issue) carried out during the 1988 and 1990 martian oppositions have shown that, at the $100-150 \mathrm{~km}$ spatial resolution, more than $95 \%$ of the variance in Mars spectra can be modeled using two linear spectral endmembers (bright and dark regions). Within the remaining 5\%, distinct spatially coherent units are detected which correlate with ices, condensates, different degrees of alteration and compositional heterogeneities within the dark terrains. The interpretation leads to a global description of the surface state of alteration consistent with the spatial distribution of the bright regions being more altered than the dark. Within the less altered regions, $\mathrm{Fe}^{2+}$ crystal field absorption bands are detected, indicative of the presence of mafic minerals in agreement with a crustal basaltic composition. These features are the most conspicuous in the volcanic regions of Syrtis Major and Hesperia Planum. Finally, a systematic survey of the Tharsis region, including the transition region toward the southern dark terrains, has shown a progressive modification of the spectra morphology when crossing the transition zone, in possible relationship with the global geomorphic dichotomy (Martin et al. 1994). Spectro-imaging orbital observations obtained by Phobos-2 covered 9 low latitude zones and have confirmed these results (Bibring et al. 1990). In addition, examination of reflectance spectra obtained across Syrtis Major plateau has narrowed the range of composition to augite-bearing basalts (Mustard et al. 1993). Also, it has provided new information about the composition and surface variability within the bright soil regions (Murchie et al., 1993). Most bright soil exhibits a narrow absorption features near $2.2 \mu \mathrm{m}$ comparable to those identified in Hawaiian palagonites, suggesting the presence of poorly crystalline phyllosilicate. The $3 \mu \mathrm{m} \mathrm{H}_{2} \mathrm{O}$ band also exhibits spatially coherent variations which may result from differences in the content of molecular water in soils developed on different deposits. Additionally, the position and shape of the $0.9 \mu \mathrm{m} \mathrm{Fe}{ }^{3+}$ absorption indicate hematite to be present in bright soils of many areas, including Tharsis. However, other ferric minerals are also detected. This analysis reveals that the bright martian soil is compositionally diverse and not fully mixed by eolian activity. It may result from different histories of chemical alteration of parent materials and derived soils, in relation to local effects of liquid water (Murchie et al. 1993).

\subsection{Earth}

Taking the same remote sensing approach and looking back to the Earth from airborne observations, the surface appears tremendously more complex in terms 
of spectroscopy than the lunar or martian surfaces, both because of the existence of a very evolved surface geology and of the intrinsic environmental variability related to the hydrosphere/atmosphere. It is probably one of the future major challenges for imaging spectroscopy to decipher and monitor this "jungle" of spectroscopic information. For this purpose, advanced remote sensing methodologies are being developed which will replace soon the limited methods of classification currently in use. These new coming approaches are based on multiple mixing modelling techniques (e.g., Sabol et al. 1993; Chabrillat et al. 1993). As an example, such a methodology is applied for the lithological discrimination across a peridotite massif located in southern Spain. Basically, iterative spectral mixture analyses are performed with the objective of : (i) identifying the geological target in the hyperspectral image, (ii) discriminating within the target the subtle mineralogical variations under study, which were previously hidden because of the high variability of the background. It shows that spatially coherent mineralogical information can be mapped within the massif, despite the presence of sparse vegetation and the fact that the spatial scale of the airborne analysis is much larger ( 20 to $100 \mathrm{~m}$ pixel size) than the one-to-ten meters scale, at which mineralogical heterogeneities are observed on the field.

\section{Conclusion}

The different cases briefly described here concerning the study of solid planetary surfaces demonstrate the wealth of the 3D-spectroscopy approach in this particular field. Coupled with advanced methodologies concerning the data analysis, it is presently revolutionizing the remote sensing world of planetary sciences.

\section{References}

Adams, J.B., Smith, M.O., \& Johnson, P.E. (1986), J. Geoph. Res., 91, 8113. Bell, J.F. III, \& al.(1990), Proc. Lun. Planet. Sci. Conf. XXth, 479.

Bell, JF. III (1992), Icarus, 100, 575.

Bibring, J.P., \& al. (1990), Proc. Lunar Planet. Sci. Conf. XXth, 461.

Chabrillat, S., \& al. (1993), Actes du Colloque "Changements d'ffiéchelle dans les modffièles de l'environnement et de la télédétection", Strasbourg, 37.

Head, J.W., \& al. (1993), J. Geoph. Res., 98, no E9, 17149.

Martin, P., \& al. (1994), Lun. Planet. Sci. Conf. XXVth, 837.

Murchie, S., \& al. (1993), Icarus, 105, 454.

Mustard, J.F., \& al. (1993), J. Geoph. Res., 98, no E2, 3387.

NASA (1987). HIRIS: High-Resolution Imaging Spectrometer, vol. IIc, 74p.

Pieters, C. M., \& Wilhelms, D.E. (1985), J. Geoph. Res., 90, C415.

Pinet, P.C., \& Chevrel, S. (1990), J. Geoph. Res., 95, no B9, 14435.

Pinet, P.C., Chevrel, S., \& Martin, P. (1993), Science, 260, 797.

Sabol, D.E., \& al. (1993), JPL Publication 93-26, 1, 157. 